\title{
EVALUATION OF HEMATOLOGY CONSULTATIONS IN COVID-19 PANDEMIC
}

\author{
Atakan Tekinalp ${ }^{1}$, Sinan Demircioğlu ${ }^{1}$, Hatice Zeynep Dikici ${ }^{1}$, and Ozcan Ceneli ${ }^{1}$ \\ ${ }^{1}$ Necmettin Erbakan University Meram Medical Faculty Hospital
}

October 1, 2020

\begin{abstract}
This study aims to evaluate the COVID-19 pandemic consultations requested from the division of hematology in the hospital. We recorded the following features of the patients: demographic information, the status of outpatient or inpatient, the clinic requested consultation, the result of the consultation, having a hematological disease, diagnosis and COVID-19 suspicion. In this study, 82 cases were evaluated. The median age of the patients was 49,5. Females were mild higher (56,1\% vs. 43,9\%). Eight of the patients was suspected with COVID-19. The frequency of suspicion in patients with the previously diagnosed hematological disorder was higher significantly than patients without previously diagnosed $(42,9 \%$ vs. $6,7 \% \mathrm{p}=0,018)$. The frequency of suspicion was higher significantly inpatients than outpatients $(19,4 \%$ vs. $2,2 \%, \mathrm{p}=0,019)$. Only two of the patients who was suspected with COVID-19 were PCR-positive and these patients were diagnosed with concomitant hematologic malignancy; CLL and MCL. The most common clinic and cause of this consultation of this clinic were gynecology and thrombocytopenia, respectively, and most of the patients were diagnosed with pseudothrombocytopenia. After the consultation for all patients, the diagnosis was drug-related cytopenia; however, detailed evaluation for cytopenia was delayed to period which after the pandemic because any patients had no deep levels of cytopenia. Although our study has some limitations, this study is remarkable because this is the first study that has investigated hematology consultation during the pandemic. We think that more significant results will be achieved as new data become available, as long as the pandemic continues.
\end{abstract}

\section{INTRODUCTION}

Some patients with unexplained viral pneumonia were reported in Wuhan, a city in the Hubei Province of China, at the end of 2019. Following its identification as a novel coronavirus pathogen (SARS-CoV-2) and an increasing number of cases in many countries, especially in Europe, World Health Organization (WHO) declared the COVID-19 as a pandemic on March 11, 2020. ${ }^{1}$ In this process, many data show that COVID-19 should be considered as a systemic disease, including respiratory, neuromuscular, cardiovascular, dermatological, immune and hematological systems. ${ }^{2,3}$ Therefore, in addition to treatment guidelines of COVID-19, various management guidelines related to specific divisions have been published from diverse societies and centers. ${ }^{4-6}$

Some studies reported that patients who were diagnosed with cancer and also immunosuppressed have a significantly higher risk of infection with SARS-CoV-2. ${ }^{7,8}$ Although the infection does not have any specific clinical findings, several hematological findings, such as lymphopenia, thrombocytopenia, prolonged coagulation tests, increased fibrin degradation products and increased ferritin levels, were also defined in patients who are virus-infected. ${ }^{5}$

To our knowledge, there was no study evaluated hematology consultation in the COVID-19 era in the literature. Herein, we aimed to present features of patients who consulted the hematology unit in this term.

\section{METHOD}


This study was designed as a retrospective study. Patients consulted hematology unit at Necmettin Erbakan University, Meram Medical Faculty, from March 14, 2020 to May 14, 2020 were evaluated in this study. We recorded patients' characteristics, such as demographic (age, gender), the status of outpatient or inpatient, the clinic requested consultation, reason and result of the consultation, having a hematological disease or not, diagnosis and COVID-19 suspicion. Our study was approved by the Ethics Committee of the Commission for Scientific Research of the Ministry of Health of the Republic of Turkey and the Ethics Committee of Meram Medical Faculty.

\section{Statistical Analysis}

Data were analized using the IBM SPSS program (version 22.0). Distribution for continuous data was evaluated using the Kolmogorov-Smirnov test. Median (minimum and maximum values) and percentage were used for descriptive statistics. The comparison of the categorical variables was performed using Chisquare test. The p-value of less than 0,05 was accepted as statistically significant.

\section{RESULTS}

From March 14, 2020 to May 14, 2020, we evaluated 82 cases who consulted the adult hematology unit. The median age of the patients was 49,5 (18-93). Forty-six $(56,1 \%)$ of the patients were female and 36 of the patients $(43,9 \%)$ were males. The number of the consultation requested from outpatients was $46(56,1 \%)$, while the inpatients were $36(43,9 \%)$. The clinic that requested the most consultation was gynecology with 17 of patients $(20,7 \%)$. Distribution of the clinics requested a consultation is listed in Table 1.

Eight patients were suspected with COVID-19 because of clinical signs, such as fever, cough and headache. Only two of these patients had COVID-positive according to PCR results. The frequency of suspicion of the COVID-19 was statistically significantly higher in patients with previously hematological diagnosed and also in consultation requested from inpatients $(\mathrm{p}=0,018$ and $\mathrm{p}=0,019$, respectively). Table 2 shows distribution associated with suspected COVID-19 patients according to features of the hematological disease and outpatient or inpatient.

Seven of the patients previously hematologic diagnosed were Hodgkin's lymphoma, chronic lymphocytic leukemia (CLL), myelodysplastic syndrome (MDS), idiopathic thrombocytopenic purpura (ITP), multiple myeloma, Bernard- Soulier syndrome and hereditary spherocytosis with one patient in each of them. In the other 75 patients, any clinical pathologic features were not found in 11 patients and diagnostic evaluation had not been finished yet in 12 patients. The most common diagnosis was drug-related cytopenia with 11 patients $(20,4 \%)$ of the 54 patients. Distribution of diagnoses is presented in Table 3. The less common diagnoses were presented like "the other" with one patient each of the diagnoses. In Table 3, diagnosis of Budd-Chiari syndrome, portal ven thrombosis, retinal ven thrombosis and hereditary thrombophilia were entitled under the upside of "thrombosis".

\section{DISCUSSION}

Recently, COVID-19 infection, declared as a pandemic, has been influencing many clinical practising and daily-life. Some hospitals have been begun to serve for only COVID-19 infected patients except for patients who had recourse to emergency service, hematology and oncology clinics and also some elective surgical operations were delayed in our country. In this study, we aimed to evaluate hematology consultation requested in such a pandemic period.

In our study, we reported 82 patients during two months in the pandemic. The mean age was 49,5 (18-83), which was consistent with James's study published in $2015 .^{9}$

The risk of COVID-19 infection is higher in immunocomprimised patients and cancer patients who received chemotherapy. ${ }^{7}$ In a retrospective cohort, Fox et al. reported 55 patients with hematological disorders and COVID-19 infection who were tested using PCR or diagnosed with based on radiological findings. ${ }^{10}$ Only eight patients was suspected with COVID-19 in our study, and the frequency of suspicion in patients with the previously diagnosed hematological disorder was $42,9 \%$, which was higher significantly compare to patients 
without previously diagnosed $(\mathrm{p}=0,018)$. On the other hand, the frequency of suspicion of COVID-19 in in-patients was also higher than outpatients, $19,4 \%$ and $2,2 \%(\mathrm{p}=0,019)$, respectively. However, only two of eight patients had PCR-positive. Interestingly, although a significant difference to the frequency of suspicion of COVID-19 was not found between patients new hematologic diagnosed and others, COVID-19 infection was diagnosed concomitant a new hematologic malignancy, CLL and MCL, in both PCR-positive patients. These data show that the findings of COVID-19 may be similar to findings of hematologic malignancies.

In our study, patients previously diagnosed hematologic disorder were not diagnosed with COVID-19 infection although $\mathrm{He}$ et al. reported that as high as $10 \%$ of patients with hematologic malignancies were COVID-19 infected in a retrospective cohort. ${ }^{11}$ We explain this paradox as follows. We evaluated only consultations and only the early period of pandemic in this study. Additionally, in our university hospital, during the pandemic, most of the clinics have been utilized as a COVID-19 clinic and hematologist and some of the assistants in the internal medicine have been worked in these clinics. Thereby, formal consultation from some of the patients may not have requested.

Many clinics request hematology consultation in daily practice. Gynecology is the most common clinic requested consultation in our study. The most cause of these consultations was thrombocytopenia, one of the common problems during pregnancy in 12 patients. In a retrospective series, Parnas et al. reported that gestational thrombocytopenia occurs $59 \%$ of the 199 pregnant women. ${ }^{12}$ However, the frequency of gestational thrombocytopenia in our study was $41 \%$. The lower ratio may be due to a low number of patients and some ethological tests in some patients having not been completed yet. Causes of other consultations requested from gynecology were anemia, splenomegaly and to discuss for using low molecular weight heparin. Anemia and splenomegaly were found to be associated with pregnancy as a result of the etiological evaluation.

As seen in Table 3, drug-related cytopenia is found as the most common diagnosis. Of the 11 patients with cytopenia, six were neutropenic, three were thrombocytopenic, and two were both. Because any patient had not a deep level of cytopenia, a detailed evaluation was delayed to period which after the pandemic and taking of the suspect drug was discontinued only. These consultations were requested with the following clinics: oncology, psychiatry, rheumatology and neurology. Oncology is one of the clinics; the second common requested consultation in our study. Thrombocytopenia, anemia, lymphadenopathy and leukocytosis were causes of the consultation. Particularly drug related-thrombocytopenia may be a significant reason to discontinue chemotherapy. Ten Berk et al. reported that the frequency of chemotherapyinduced thrombocytopenia is $3 \%$ to $4 \%$ and patients received platelet transfusion are less than $3 \% .{ }^{13}$ Severe thrombocytopenia is not usual in also COVID-19 infection. It was reported that only $5 \%$ of patients had thrombocyte lower than $100.000 / \mu \mathrm{L}$, in a case series, in Wuhan, whereas lymphopenia was the most common blood count abnormality in COVID-19 infection. Leukopenia may be determined in 33,7\% of patients. ${ }^{14-16}$ However, leukopenic and thrombocytopenic patients had no symptoms and signs of COVID-19, so they were not tested by PCR.

COVID-19 infection may lead to a hypercoagulable and dyscoagulable situation, especially in patients with severe infection. Elevated D-dimer levels and fibrinogen can be distinguished and prognostic. ${ }^{17,18}$ Four patients with thromboembolic disorder in our study had not suspicion of COVID-19. Thus, they were researched for only their own disease.

The limitation of our study may be the absence of comparing between the COVID-19 era and the prepandemic period and the existence of 12 patients whose evaluation has not been completed yet. However, our study is crucial because, to our knowledge, there is not any study that has investigated hematology consultation during the COVID-19 pandemic in the literature.

Patients are referred to hematologist due to suspicion of hematologic disease for various symptoms and findings in daily practice, although many hematologic disorders are rare. Because of similarities in symptoms and signs, COVID-19 related hematologic complications and applying to hospital by non-COVID-19 causes; we think that hematology consultations will continue, as long as the pandemic continues.

\section{References:}


1. Lu R, Zhao X, Li J, et al. Genomic characterisation and epidemiology of 2019 novel coronavirus: implications for virus origins and receptor binding. Lancet. 395: 565-574, 2020.

2. Mehta P, McAuley DF, Brown M, et al. COVID-19: consider cytokine storm syndromes and immunosuppression. Lancet 395: 1033-1034, 2020.

3. Driggin E, Madhavan MV, Bikdeli B, et al. Cardiovascular Considerations for Patients, Health Care Workers, and Health Systems During the Coronavirus Disease 2019 (COVID-19) Pandemic. J Am Coll Cardiol 2020 Mar 18. doi: 10.1016/j.jacc.2020.03.031

4. Darlenski R, Tsankov N. COVID-19 pandemic and the skin: what should dermatologists know? Clin Dermatol 2020 Mar 24. https://doi.org/10.1016/j.clindermatol.2020.03.012

5. Weinkove R, McQuilten ZK, Adler J et al. Managing haematology and oncology patients during the COVID-19 pandemic: interim consensus guidance. Med J Aust 2020 May 13. doi: 10.5694/mja2.50607

6. Amanda C. Guidon, Anthony A. Amato. COVID-19 and neuromuscular disorders. Neurology 2020 Apr 13. doi:10.1212/WNL.0000000000009566

7. Liang W, Guan W, Chen R et al. Cancer patients in SARS-CoV-2 infection: a nationwide analysis in China. Lancet Oncol 21: 335-7, 2020.

8. Yu J, Ouyang W, Chua MLK et al. SARS-CoV-2 transmission in patients with cancer at a tertiary care hospital in Wuhan, China. JAMA Oncol 6(7): 1108-1110, 2020.

9. James AH, Cooper DL, Paidas MJ. Hemostatic assessment, treatment strategies, and hematology consultation in massive postpartum hemorrhage: results of a quantitative survey of obstetriciangynecologists. Int J Womens Health 4(7): 873-881, 2015.

10. Fox TA, Troy-Barnes E, Kirkwood AA et al. Clinical outcomes and risk factors for severe COVID-19 infection in patients with haematological disorders receiving chemo- or immunotherapy. Br J Haematol 2020 Jul 17. doi: 10.1111/bjh.17027

11. He W, Chen L, Chen L et al. COVID-19 in persons with haematological cancers. Leukemia 34: $1637-1645,2020$.

12. Parnas M, Sheiner E, Shoham-Vardi I et al. Moderate to severe thrombocytopenia during pregnancy. Eur J Obstet Gynecol Reprod Biol 128(1-2):163-168, 2006.

13. Ten Berg MJ, van den Bemt PM, Shantakumar S et al. Thrombocytopenia in adult cancer patients receiving cytotoxic chemotherapy: results from a retrospective hospital-based cohort study. Drug Saf 34: 1151-1160, 2011.

14. Huang C, Wang Y, Li X et al. Clinical features of patients infected with 2019 novel coronavirus in Wuhan, China. Lancet 395: 497-506, 2020.

15. Fan BE, Chong VCL, Chan SSW et al. Hematologic parameters in patients with COVID-19 infection. Am J Hematol 2020 Mar 04. doi:https://doi.org/10.1002/ajh.25774.

16. Terpos E, Ntanasis-Stathopoulos L, Elalamy I et al. Hematological findings and complications of COVID-19. Am J Hematol 95: 834-847, 2020.

17. Zhou F, Yu T, Du R et al. Clinical course and risk factors for mortality of adult inpatients with COVID-19 in Wuhan, China: a retrospective cohort study. Lancet 395: 1054-1062, 2020.

18. Han H, Yang L, Liu R et al. Prominent changes in blood coagulation of patients with SARS-CoV-2 infection. Clin Chem Lab Med 2020 Jun 25. doi:https://doi.org/10.1515/cclm-2020-0188.

Table-1: Distrubition of clinics requested consultation from hematology.

Clinic Requested

Gynecology-Obstetric General Surgery Oncology Gastroenterology Cardiology Orthopedics Psychiatry Enfection Disease Cl 


\begin{tabular}{|c|c|c|c|}
\hline $\begin{array}{l}\text { Table-2: Distrubition } \\
\text { ascociated with } \\
\text { COVID suspicion of } \\
\text { patients to } \\
\text { hematological disease } \\
\text { and root of patients. }\end{array}$ & $\begin{array}{l}\text { Table-2: Distrubition } \\
\text { ascociated with } \\
\text { COVID suspicion of } \\
\text { patients to } \\
\text { hematological disease } \\
\text { and root of patients. }\end{array}$ & $\begin{array}{l}\text { Table-2: Distrubition } \\
\text { ascociated with } \\
\text { COVID suspicion of } \\
\text { patients to } \\
\text { hematological disease } \\
\text { and root of patients. }\end{array}$ & $\begin{array}{l}\text { Table-2: Distrubition } \\
\text { ascociated with } \\
\text { COVID suspicion of } \\
\text { patients to } \\
\text { hematological disease } \\
\text { and root of patients. }\end{array}$ \\
\hline & $\begin{array}{l}\text { COVID19 Suspicion } \\
\text { YES, n }(\%)\end{array}$ & $\begin{array}{l}\text { COVID19 Suspicion } \\
\text { NO, n }(\%)\end{array}$ & $\mathrm{P}$ \\
\hline $\begin{array}{l}\text { Previously } \\
\text { Hematologic Disorder } \\
\text { Yes, n:7 } \% \text { No. n:75 (\%) }\end{array}$ & $3(42,9) 5(6,7)$ & $4(57,1) 70(93,3)$ & $0,018^{*}$ \\
\hline $\begin{array}{l}\text { New, n:7 Hematologic } \\
\text { Diagnosed (After } \\
\text { Consultation) Yes, n:18 } \\
(\%) \text { No, n:64 (\%) }\end{array}$ & $4(22,2) 4(6,3)$ & $14(77,8) 60(93,8)$ & $0,06^{*}$ \\
\hline $\begin{array}{l}\text { Root of Patient } \\
\text { Inpatient, n:36 (\%) } \\
\text { Outpatient, n:46 (\%) }\end{array}$ & $7(19,4) 1(2,2)$ & $29(80,6) 46(97,8)$ & $0,019^{*}$ \\
\hline $\begin{array}{l}\text { Fisher's Exact } \\
\text { qi-square } 8(9,8) 74 \\
(90,2)\end{array}$ & $\begin{array}{l}\text { *Fisher's Exact } \\
\text { qi-square } 8(9,8) 74 \\
(90,2)\end{array}$ & $\begin{array}{l}\text { *Fisher's Exact } \\
\text { qi-square } 8(9,8) 74 \\
(90,2)\end{array}$ & $\begin{array}{l}\text { *Fisher's Exact } \\
\text { qi-square } 8(9,8) 74 \\
(90,2)\end{array}$ \\
\hline
\end{tabular}

Table-3: Distrubition of new diagnosis after consultation

Diagnosis

Drug-related cytopenies Gestationel thrombocytopenia Iron deficiancy anemia Thrombosis Enflamation anemia Secondary 